This information is current as of April 26, 2023.

\title{
Diffusion-Weighted MR Imaging in Posterior Ischemic Optic Neuropathy
}

Ashish Verma, Krishan K. Jain, Suvash Mohan and Rajendra V. Phadke

AJNR Am J Neuroradiol 2007, 28 (10) 1839-1840

doi: https://doi.org/10.3174/ajnr.A0710

http://www.ajnr.org/content/28/10/1839 


\section{Diffusion-Weighted MR Imaging in Posterior Ischemic Optic Neuropathy}

We read with interest the case report by Mathur et $\mathrm{al}^{1}$ in which they have described an acute ischemic event in the posterior part of the intraorbital optic nerve consequent to rhinocerebral mucormycosis and its early detection by diffusion-weighted MR imaging (DW-MR imaging). In this regard, we would like to report another similar case that we came across in our tertiary care institute and benefited from the above report.

In April 2007, a 40-year-old man with diabetes presented to the emergency department with sudden-onset holocranial headache associated with pain in the left eye and diplopia. There was associated proptosis of the left eye. The patient was referred to us for MR imaging of the brain, which showed a normal brain parenchyma and intracranial venous sinuses (as seen on MR venogram). On MR angiogram (Fig 1A) there was nonvisualization of the left internal carotid artery (ICA) with reformation of the left middle and anterior cerebral arteries via the collateral circulation from the right side. Subtle increase in the caliber of the left cavernous sinus compared with the right was noted; no direct or indirect evidence of cavernous sinus thrombosis was noted. Enhancing soft tissue filling the whole of the left sinonasal area was incidentally seen. However, no direct intracranial extension of a sinonasal pathologic process was noted. Edema was seen involving the left lateral rectus muscle (Fig $1 B$ ). DW-MR imaging in the axial and sagittal planes with 3-mm sections was done, which showed restriction in the posterior part of the intraorbital segment of the optic nerve on apparent diffusion coefficient (ADC) maps $\left(0.635 \times 10^{-3}\right.$ $\left.\mathrm{mm}^{-2} / \mathrm{s}\right)$. Increased ADC $\left(1.7 \times 10^{-3} \mathrm{~mm}^{-2} / \mathrm{s}\right)$ suggesting a vasogenic edema was noted in the anterior part. The posterior part of the nerve did not show alteration in signal intensity on fat-saturated T2-weighted and gadolinium-enhanced, fat-saturated T1weighted sequences. Fundus examination was normal. This led to a diagnosis of acute posterior ischemic optic neuropathy (PION). The possibility of developing optic atrophy and risks of thromboembolism were explained, and the patient was managed conservatively. Histopathologic specimens from scrapings from the nasal cavity suggested the possibility of Aspergillus infection. The ophthalmic examination showed positive perception of light and projection of rays. However, adequate perimetry was not possible because of poor patient cooperation. Follow-up screening revealed edema in the whole of the intraorbital optic nerve, even in the fat-saturated T2-weighted sequence. The patient sustained multiple territorial infarcts of varying sizes in the subsequent days of his hospital stay, which suggested an embolic phenomenon. He died of a cardiac arrest on day 11 .

In most cases of fungal sinusitis, PION is caused by occlusion of the central retinal artery, ${ }^{2}$ necrosis of the ophthalmic artery, ${ }^{3}$ infarction and necrosis of the optic nerve, ${ }^{2,3}$ or direct infection of the optic nerve by mucormycosis. ${ }^{3}$ In the absence of direct invasion, a sinonasal infection can cause ipsilateral cavernous sinus phlebitis via the pterygoid plexus of the veins, thrombosis of the internal carotid artery being secondary to involvement of the cavernous sinus. Again, isolated ischemia in the posterior part of the intraorbital optic nerve, without direct involvement by a sinus pathologic process, could result from thrombosis of the vessels supplying the perineural pial plexus that is the principal source of blood supply to this segment. ${ }^{4,5}$ The perineural plexus supplying the posterior part of the optic nerve is formed by a recurrent branch from the anterior superior hypophyseal artery, branches from the ophthalmic artery, and the intraneural recurrent branch from the central artery of the retina, all originating from the segment of the ICA, which was thrombosed in our patient. ${ }^{4}$ Anastomosis between the orbital branches of the ICA and the facial branches of the external carotid artery may help feed the anterior two thirds of the optic nerve by retrograde flow in the event of occlusion of the ICA, the posterior third remaining deprived.

No treatment of proved value is available for cases having direct extension of infection, yet a trial of systemic corticosteroids can help improve vision in a minority of patients. ${ }^{6}$ In cases like ours, where arterial thrombosis was the underlying cause, thrombolysis may be helpful. In both situations, DW-MR imaging guides early institution of appropriate therapy and must be considered part of a routine imaging protocol.
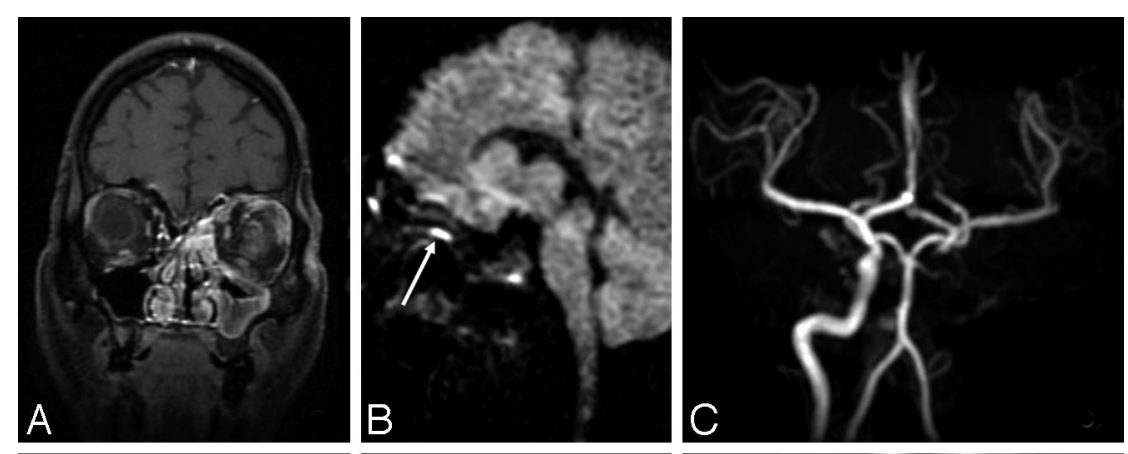

Fig 1. MR imaging shows enhancing mucosal thickening in the left sinonasal complex on coronal postcontrast fat-saturated T1-weighted $(A)$ and axial T2-weighted sequences $(B)$. In addition, the T2-weighted image shows a bulky, enhancing left cavernous sinus with nonvisualization of left ICA flow void (suggesting thrombosis). A 3D time-of-flight MRA (C) shows absence of the left ICA and paucity of the branches of the left distal MCA. Sagittal $(D)$ and axial $(E)$ DW images show restriction in the posterior part of the intraorbital optic nerve (arrow). The ADC map (F) shows reduced ADC in the posterior part (arrow) and increased ADC in the anterior part, respectively.
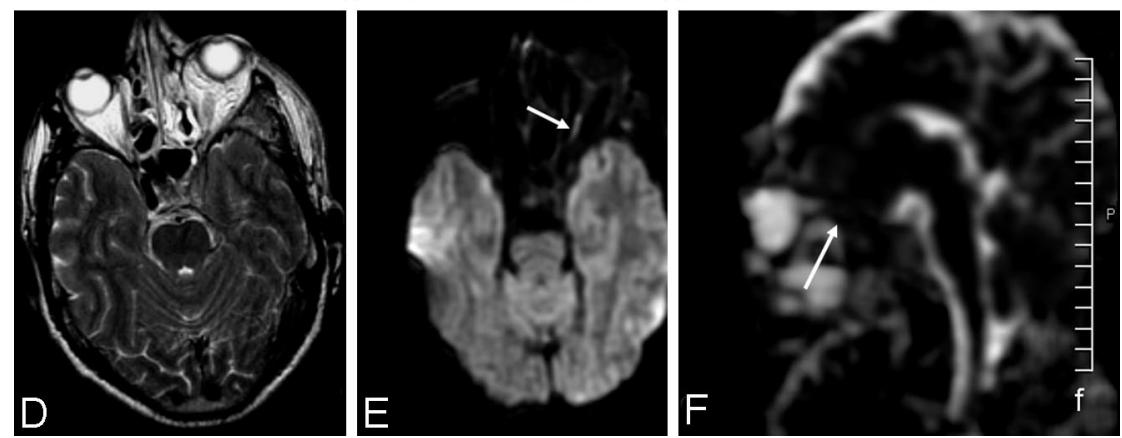


\section{References}

1. Mathur S, Karimi A, Mafee MF. Acute optic nerve infarction demonstrated by diffusion-weighted imaging in a case of rhinocerebral mucormycosis. AJNR Am J Neuroradiol 2007;28:489-90

2. Ferry AP, Abedi S. Diagnosis and management of rhino-orbitocerebral mucormycosis (phycomycosis). A report of 16 personally observed cases. Ophthalmology 1983;90:1096-104

3. Downie JA, Francis IC, Arnold JJ, et al. Sudden blindness and total ophthalmoplegia in mucormycosis. A clinicopathological correlation. J Clin Neuroophthalmol 1993;13:27-34

4. Blunt MJ, Steele EJ. The blood supply of the optic nerve and chiasma in man. J Anat 1956;90:486-93

5. Al-Shafai LS, Mikulis DJ. Diffusion MR imaging in a case of acute ischemic optic neuropathy. AJNR Am J Neuroradiol 2006;27:255-57

6. Hayreh SS. Ischaemic optic neuropathy[published erratum appears in Indian J Ophthalmol 2000;48:317]. Indian J Ophthalmol 2000;48:171-94

Ashish Verma

Krishan K. Jain

Suvash Mohan

Rajendra V. Phadke

Department of Radiodiagnosis

Sanjay Gandhi Post Graduate Institute of Medical Sciences

Lucknow, India

DOI 10.3174/ajnr.A0710

\section{Cerebral Aneurysms in a Patient with Osteogenesis Imperfecta and Exon 28 Polymorphism of COL1A2}

Petruzzellis et $\mathrm{al}^{1}$ present an interesting case of a patient with osteogenesis imperfecta and a ruptured aneurysm at the fenestrated basilar artery. However, they misidentify the fenestration as a vertebral fenestration and, as such, do not seem to relate the fenestration to the basilar aneurysm. Figures $1 A$ and $2 A$ beautifully show well-known features of the basilar fenestration just above the vertebral junction ${ }^{2,3}$ : the joining of both vertebral arteries, subsequent division of the basilar artery into 2 arms, effective widening of the distance between the lateral walls of both arms compared with the basilar diameter beyond, and rejoining of the fenestrated arms into 1 artery.

The relationship of aneurysms of the proximal basilar trunk and basilar fenestrations is well known. ${ }^{2,3}$ A substantial series by Campos et $\mathrm{al}^{2}$ of 59 aneurysms of the basilar trunk found $35.5 \%$ in association with definite fenestrations, all but 1 at the proximal end of the fenestration. It is possible that other fenestrations were there but were not discerned because the aneurysms were superimposed over the basilar fenestrations, with the result of a higher incidence. With easy-to-do maximum intensity projections or multiplanar reformations with high resolution on CTA or MRA, ${ }^{4}$ viewed with a high index of suspicion, we can now readily show fenestrations. With sectioning of image datasets, aneurysms will less likely superimpose fenestrations.

In the case report by Petruzzellis et al, ${ }^{1}$ the discussion of osteogenesis imperfecta is interesting and educational for that entity. However, by not paying attention to the details of their own images, they missed the real point of this case. The important entity of aneurysm at the basilar fenestration is considered to develop as a result of hemodynamic forces on the "crotch" of the fenestration, leading to aneurysms in patients without osteogenesis imperfecta. In this patient with osteogenesis imperfecta and a fenestration aneurysm, the question raised is whether osteogenesis imperfecta is an innocent coincidental bystander.

The authors claim that the aneurysm seen 4 months after coiling is new, with angiograms showing a difference between the right posterior oblique views in Fig 1 and left posterior oblique, lateral, and Towne views in Fig 2. Again, the lack of attention to detail of these images leads the authors to claim that a new aneurysm developed in 4 months. This conveniently shows the neck of the so-called "new aneurysm" in the same spot as the treated aneurysm, just at the left side of the proximal split of the basilar fenestration. We can compare Fig $1 A$ with Fig $2 C$ for the closest possible orientation, and this comparison gives strong suggestion of the same aneurysm with a refilled neck after coiling, a common enough finding. It seems, then, that this aneurysm is not a rare, newly developed one but another occurrence of lack of attention to the details of the case.

Many reports describing coiling of aneurysms at the basilar fenestration are in the literature. ${ }^{5}$ Perhaps this is the first reported case in a patient with osteogenesis imperfecta, but the discussion in this case avoids this main theme through oversight of important findings and claims others that are dubious. The American Journal of Neuroradiology has an educational responsibility to show readers exemplary neuroimaging cases and interpretations, in addition to rigorous scientific reports and interesting musings of authors in discussion.

\section{References}

1. Petruzzellis M, De Blasi R, Lucivero V, et al. Cerebral aneurysms in a patient with osteogenesis imperfecta and exon 28 polymorphism of COL1A2. AJNR Am J Neuroradiol 2007;28:397-98

2. Campos J, Fox AJ, Viñuela F, et al. Saccular aneurysms in basilar artery fenestration. AJNR Am J Neuroradiol 1987;8:233-36

3. Uda K, Murayama Y, Gobin YP, et al. Endovascular treatment of basilar artery trunk aneurysms with Guglielmi detachable coils: clinical experience with 41 aneurysms in 39 patients. J Neurosurg 2001;95:624-32

4. Bharatha A, Fox AJ, Aviv RI, et al. CT angiographic depiction of a supraclinoid ICA fenestration mimicking aneurysm, confirmed with catheter angiography. Surg Radiol Anat 2007;29:317-21

5. Saatci I, Cekirge HS, Karcaaltincaba M, et al. Endovascular treatment of kissing aneurysms at the fenestrated basilar artery. Case report with literature review. Surg Neurol 2002;58:54-58; discussion 58

$$
\begin{array}{r}
\text { Allan Fox } \\
\text { Sean Symons } \\
\text { Richard Aviv } \\
\text { Department of Neuroradiology } \\
\text { Sunnybrook Sciences Center } \\
\text { Toronto, Ontario } \\
\text { Canada }
\end{array}
$$

DOI 10.3174/ajnr.A0727

\section{Reply:}

We thank Dr. Fox for his comments concerning our previously published letter to the editor entitled, "Cerebral Aneurysms in a Patient with Osteogenesis Imperfecta and Exon 28 Polymorphism Of COL1A2." ${ }^{1}$ He strongly informs us of a misunderstanding regarding the de novo aneurysm that developed after 4 months. Dr. Fox points out some potential errors in our diagnosis, claiming that the de novo aneurysm is actually a refilling of the previously treated one. We believe that indeed at 4 months, our images revealed that a new aneurysm had developed in front of the previous one (Fig $2 B$ ). Further evidence of this was found in the posttreatment un-subtracted images (not published due to space constraints), in which it was possible to appreciate the stent crossing the vertebrobasilar junction and the coils occluding 2 different and clearly separable aneurysms.

Finally, we are grateful to Dr. Fox for bringing to our attention some bibliographic references that may help us achieve a better understanding of the anatomic features of this particular case. 